\title{
Methicillin-resistenter Staphylococcus aureus (MRSA) - Diagnostik
}

\author{
H. Linde \\ N. Lehn
}

\section{Methicillin-resistant Staphylococcus aureus (MRSA) - \\ diagnostics}

\section{Staphylococcus aureus}

S. aureus ist inner- und außerhalb des Krankenhauses zu einem der wichtigsten Erreger von Wund- und anderen Infektionen geworden. Eine Fülle von Pathogenitätsfaktoren kann zu den unterschiedlichsten Krankheitsbildern führen, bei denen infektiöse oder toxische Komponenten eine unterschiedliche Bedeutung haben (Tab.1). Von klinisch besonderer Bedeutung bei schweren Infektionen ist die scheinbar paradoxe Eigenschaft des Erregers, sich gleichzeitig in Abszessen abzukapseln und sich systemisch auszubreiten. Ebenfalls hervorzuheben ist die hohe Affinität zu implantierten Fremdkörpern mit der Gefahr für fremdkörperassoziierte nosokomiale Infektionen. Verschiedene S. aureus-Stämme unterscheiden sich in ihrer Virulenz, die vermutlich über ein komplexes Zusammenspiel einer Vielzahl dieser Faktoren entsteht $(2,6)$.

\section{S. aureus mit Panton-Valentine-Leukozidin}

Eine Besonderheit sind toxinvermittelte Krankheitsbilder, bei denen die Wirkung eines Toxins im Vordergrund steht (Tab.1, Gruppe 2). Die Bestimmung eines „Pathogenitätsprofils“ (analog dem Resistenzprofil) hat wegen der hohen Anzahl der Pathogenitätsfaktoren und der schwierigen Interpretation noch nicht Einzug in die Routinediagnostik gefunden. Neuere Daten bestätigen jedoch ältere Beobachtungen einer engen Assoziation des Panton-Valentine-Leukozidins (PV-Leukozidin) mit dem Auftreten von Abszessen und Furunkeln, sowie selten, nekrotisierenden Pneumonien (oft als Superinfektion viraler Erkrankungen; Letalität bis 70\%)(8). Häufig treten die Abszesse/Furunkel rezidivierend und ohne erkennbare Eintrittspforte auf. Verläufe über Jahre kommen vor. Ebenfalls charakteristisch sind Häufungen unter Personen mit engem körperlichem Kontakt (Sexualpartner, Familienmitglieder, Ringer, Gefängnisinsassen, Schiffs-
Tab. 1 Durch S. aureus verursachte Krankheitsbilder.

\begin{tabular}{|c|c|}
\hline Gruppe 1: Eitrige Infektionen & Gruppe 2: Toxinbedingte Erkrankungen \\
\hline $\begin{array}{l}\text { Haut- und Weichteilinfektionen, } \\
\text { Impetigo, Abszesse }\end{array}$ & „Toxic-Shock“-Syndrom \\
\hline Osteomyelitis & $\begin{array}{l}\text { Bullöse Impetigo, „Staphylococcal } \\
\text { Scalded Skin“-Syndrom }\end{array}$ \\
\hline Pneumonie & Rezidivierende Abszesse \\
\hline Sepsis & Lebensmittelvergiftung \\
\hline
\end{tabular}

besatzungen, etc.). Das PV-Leukozidin bindet mit hoher Affinität an Granulozyten und Makrophagen und führt durch Porenbildung zu einer massiven Ausschüttung des Zellinhalts mit nachfolgendem Zelltod. Epidemiologische Brisanz entsteht dadurch, dass das Toxin durch Phagen auf andere S. aureus-Stämme übertragbar ist. Gleichzeitig lässt dieser Mechanismus verstehen, wie unterschiedliche Mosaike von Pathogenitätsfaktoren zustande kommen. Der Anteil von PV-Leukozidin-positiven S. aureus-Stämmen aus Patientenmaterial (mit oder ohne Methicillinresistenz) kann 2-6\% betragen (11).

\section{S. aureus mit Methicillin-/Mulli-Resistenz}

Weltweit werden immer häufiger S. aureus-Stämme mit Resistenz gegen Antibiotika isoliert. Die mutmaßlichen Hauptgründe sind ein steigender Selektionsdruck durch die vermehrte Anwendung von Antibiotika, ungenügendes hygienisches Verhalten sowie eine durch Breitbandantibiotika in ihrer Konkurrenzfähigkeit geschwächte/veränderte Normalflora. Von der Einführung einer neuen Substanzklasse bis zum Auftauchen von resistenten S. aureus-Mutanten dauerte es in der Regel lediglich 4 (!)

Institut

Institut für Medizinische Mikrobiologie und Hygiene, Universität Regensburg

Korrespondenz

Priv.-Doz. Dr. med. Hans-Jörg Linde, Prof. Dr. med. Norbert Lehn · Institut für Medizinische Mikrobiologie und Hygiene, Universität Regensburg · Franz-Josef-Strauss-Allee $11 \cdot 93043$ Regensburg

· Tel.: 0941/9446414 · Fax: 0941/9446402 · E-Mail: Hans-Joerg.Linde@klinik.uni-regensburg.de

eingereicht: $29.11 .2004 \cdot$ alkzeptiert: 18.2 .2005 


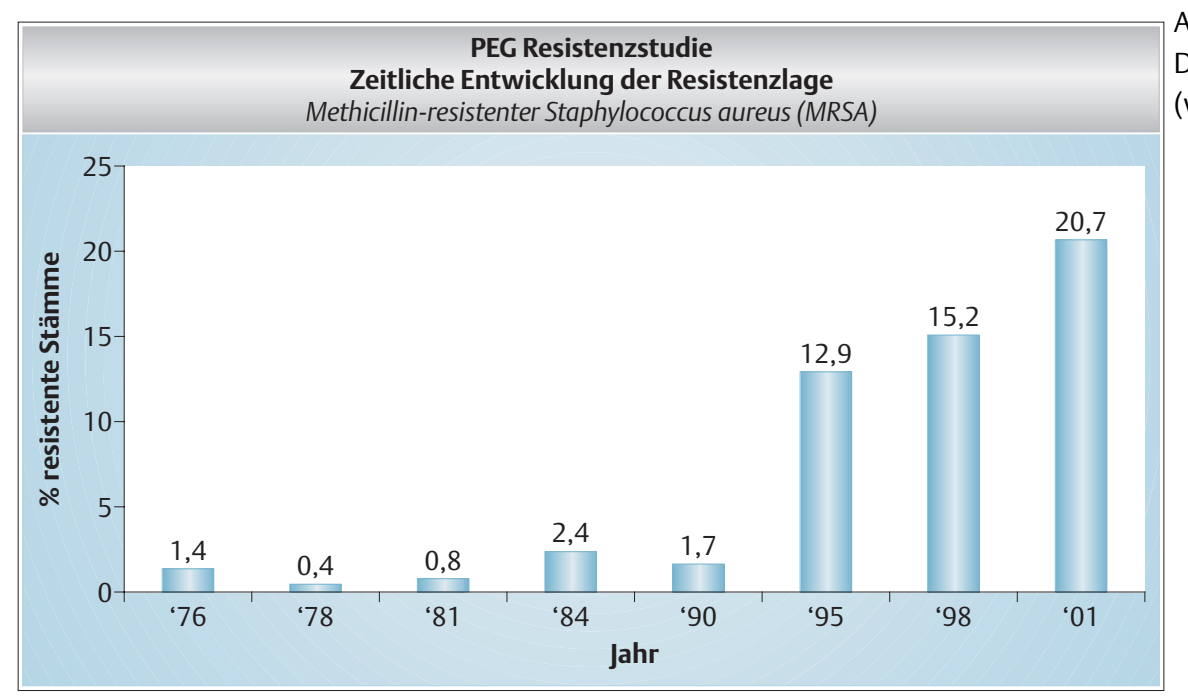

Abb. 1 Anteil von MRSA in Deutschland nach Daten der Paul-Ehrlich-Gesellschaft (www.p-e-g.de).

Jahre, mit Ausnahme von Vancomycin (20 Jahre). Seit dem ersten $S$. aureus-Isolat mit Resistenz gegen Methicillin/Oxacillin (mit Kreuzresistenz gegen alle $\beta$-Laktame durch das alternative Penizillin-Binde-Protein PBP2a, kodiert durch das mecA-Gen) im Jahr 1961 ist es weltweit zu einer bemerkenswerten Ausbreitung und Vermehrung von Methicillin-resistenten S. aureus-Stämmen (MRSA) gekommen. Zusätzlich ist eine Resistenz gegen Makrolide, Chinolone sowie Aminoglykoside bei aktuellen Isolaten aus Deutschland häufig vorhanden. Den bisherigen Gipfel der Resistenzentwicklung stellen drei unabhängig voneinander in den USA entstandene Isolate mit Vancomycinresistenz dar (VRSA), neben in Japan, den USA und Frankreich beobachteten Stämmen mit intermediärer Empfindlichkeit gegen Glykopeptide (GISA).

kurzgefasst: S. aureus kann eine Vielzahl von infektiösen, infektiös-toxischen und toxisch geprägten Krankheitsbildern auslösen. S. aureus-Stämme mit PV-Leukozidin rufen gehäuft eitrige Infektionen auch ohne erkennbare Eintrittspforte hervor. S. aureus-Stämme mit Resistenz gegen Oxacillin sind häufig auch gegen andere Antibiotikasubstanzklassen resistent und stellen therapeutisch ein Problem dar.

\section{Epidemiologie von MRSA}

Auch in Deutschland hat der Anteil von MRSA in den letzten 10 Jahren stark zugenommen. Mit regionalen Schwankungen lag der Anteil von MRSA im Landesdurchschnitt nach einer Studie der Paul-Ehrlich-Gesellschaft 2001 bei 20,7\% (Abb.1). Nach Daten des European Antimicrobial Surveillance System (EARSS) ist Deutschland zwischen 1999-2002 gleichzeitig das Land mit der größten Zunahme von MRSA in Europa. Überdurchschnittlich häufig findet sich MRSA bei Patienten nach längeren bzw. wiederholten Krankenhausaufenthalten, nach Verlegung von Intensivstationen, medizinischen Behandlungen im Ausland (vor allem Südeuropa, USA, Japan), nach Antibiotikatherapie, bei chronischen Wunden bzw. chronischen Krankheiten (z. B. Hämodialyse). Am Universitätsklinikum Regensburg erreicht die MRSA-Rate unter Risikopatienten bei Aufnahme auf Intensivstationen 25\%. Mehr als die Hälfte der während des Krankenhausaufenthalts festgestellten MRSA-Isolate
Tab.2 Risikofaktoren für MRSA und andere multiresistente nosokomiale Infektionserreger nach (12).

Antimikrobielle Therapie

Grunderkrankung: z.B. Schwere der Erkrankung, Transplantation, verlängerte Liegedauer

Verlegung in eine andere medizinische Einrichtung

Invasive Maßnahmen: Gefäßzugänge (ZVK, PVK), Urinkatheter, Intubation, Chirurgie

sind bereits bei Aufnahme nachweisbar. In Altenheimen können 2-4\% der Bewohner kolonisiert oder infiziert sein (3), vereinzelt wird über wesentlich höhere Zahlen berichtet (4). In welchem Maße sich MRSA, ausgehend von stationären medizinischen Einrichtungen über Patienten oder Personal auch in der allgemeinen Bevölkerung verbreitet, ist derzeit für Deutschland nicht ausreichend untersucht. Die im Rahmen der Einführung von DRGs erwartete Verschiebung von Leistungen aus dem stationären in den ambulanten Sektor stellt in Zukunft beim Umgang mit nosokomialen Problemkeimen neue und erhöhte Anforderungen an ambulante Einrichtungen.

Zahlen aus den Niederlanden und den skandinavischen Ländern (MRSA < $1 \%$ ) zeigen, dass eine Zunahme von MRSA in Krankenhäusern nicht unumkehrbar ist. Ob eine Umkehrung in Deutschland durch eine koordinierte Strategie (Stichworte: Diagnostik, Antibiotikaverbrauch, Standardhygiene, MRSA-spezifische Hygienemaßnahmen; s. u.) ebenfalls gelingt, wird sich vermutlich in den nächsten Jahren entscheiden und ist eine Frage von größter medizinischer Bedeutung (5). Das historische Beispiel der Ausbreitung der Penicillin-resistenten $S$. aureus vor 30-40 Jahren zeigt, dass den Keimen bei einer Zunahme in medizinischen Einrichtungen zunehmend der Sprung „hinaus“ in die Gesellschaft gelingt, und eine solche Entwicklung dann nicht umkehrbar ist.

\section{„Community-acquilred" MRSA (CA-MRSA)}

Eine Kombination von Oxacillin-Resistenz und PV-Leukozidin wurde erstmals 1998 bei S. aureus-Isolaten in den USA bei Kindern beobachtet und diese wegen des Fehlens einer Verbindung 
zu medizinischen Einrichtungen als „community-acquired“ MRSA (CA-MRSA) bezeichnet (9). Diese Stämme können die für PV-Leukozidin-positive S. aureus-Stämme typischen Krankheitsbilder verursachen, sind jedoch zusätzlich resistent gegen alle $\beta$ Laktam-Antibiotika. Auffälligerweise sind die Stämme gegen andere Antibiotikasubstanzklassen meist noch sensibel. Bei Isolaten aus Deutschland ist eine zusätzliche Resistenz gegen Fusidinsäure auffällig. Mittlerweile werden auch diese besonderen MRSAStämme weltweit isoliert. In Deutschland wurde bis vor kurzem von einem sporadischem Vorkommen ausgegangen. Aktuelle eigene Daten aus der Region Ostbayern (Regensburg) belegen jedoch ein Vorkommen seit 1995, bei seither insgesamt>250 Patienten. Eine verbesserte Diagnostik (s.u.) sollte in Zukunft mehr Wissen über die tatsächliche Verbreitung von CA-MRSA in Deutschland schaffen. Werden CA-MRSA in Krankenhäuser oder andere medizinische Einrichtungen eingeschleppt, kann dies zu nosokomialen Infektketten analog MRSA führen. Im Rahmen von zwei nosokomialen Ausbrüchen beobachteten wir bei Personal sowie bei privaten Kontaktpersonen auch Infektionen durch CAMRSA. Eine weitere Verbreitung dieser Keime hätte erhebliche Auswirkungen auf die kalkulierte Therapie von ambulant erworbenen Infektionen durch S. aureus.

kurzgefasst: Bei großen regionalen Unterschieden wird in Krankenhäusern in Deutschland derzeit eine starke Zunahme von MRSA beobachtet. Der Anteil liegt im Mittel bei $20 \%$ aller $S$. aureus-Isolate. Als neue Entwicklung werden außerhalb medizinischer Einrichtungen erworbene so genannte „community-acquired“ MRSA (CAMRSA) beobachtet, die neben der Oxacillin-Resistenz den Virulenzfaktor PV-Leukozidin besitzen.

\section{Diagnostische Verfahren}

Der Nachweis von S. aureus ist wegen des robusten, schnellen und charakteristischen Wachstums in der Regel problemlos. Schwierigkeiten ergeben sich bei antibiotischer Vorbehandlung sowie, vermutlich seltener, bei Vorliegen von „small colony variants“ (Defektmutanten mit langsamem Wachstum, u.a. bei fremdkörperassoziierten Infekten, Mukoviszidose). Zur Differenzierung und Resistenztestung stehen eine Reihe von gut evaluierten Methoden zur Verfügung (1). Zur sicheren Erkennung der Oxacillin-Resistenz müssen parallel zur weit verbreiteten Testmethode der Blättchendiffusion zusätzliche Testverfahren durchgeführt werden. Traditionelle Güteparameter wie Sensitivität/Spezifität liegen dabei im Konflikt mit dem klinischen Bedürfnis einer möglichst schnellen Diagnostik. Als Goldstandard zum Nachweis der Besiedlung des Nasenvorhofs mit MRSA gilt derzeit ein Kulturverfahren mit verlängerter Bebrütung und vorausgehendem Anreicherungsschritt (72-96 h) (13). Die Kombination von Kultur und Latexagglutination mit PBP2a-spezifischen Antikörpern kann ein Ergebnis nach 24h liefern. Demgegenüber gelingt mittels automatisierter DNA-Isolierung und Real-time PCR der Nachweis von MRSA direkt aus Nasenabstrichtupfern innerhalb 3-5h (7). Welche Methode zum Einsatz kommt, ist eine Frage der Laborausstattung, der klinischen Fragestellung sowie - vordergründig - der in Kauf genommenen Kosten. Ein Vorschlag zur Anwendung der verschiedenen
Tab.3 Diagnostische Methoden für MRSA und ihre Indikation.

\begin{tabular}{|c|c|c|c|}
\hline Methode & Zeitdauer & Indikation & Kommentar \\
\hline $\begin{array}{l}\text { PCR direkt aus } \\
\text { Probenmaterial }\end{array}$ & $3-5 h$ & $\begin{array}{l}\text { - Aufnahmescreening } \\
\text { bei Risikopatienten } \\
\text { - Grampositive Kokken } \\
\text { in Blutkultur/sterilem } \\
\text { Material }\end{array}$ & $\begin{array}{l}\text { bei elektiven } \\
\text { Eingriffen vor Auf- } \\
\text { nahme möglich }\end{array}$ \\
\hline $\begin{array}{l}\text { Kultur + Identifizie- } \\
\text { rung/PBP2a-Nach- } \\
\text { weis mittels } \\
\text { Latexagglutination }\end{array}$ & $24-48 \mathrm{~h}$ & $\begin{array}{l}\text { - Aufnahmescreening } \\
\text { bei Risikopatienten } \\
\text { - Staphylokokken } \\
\text { aus steriler } \\
\text { Entnahmestelle }\end{array}$ & \\
\hline $\begin{array}{l}\text { Kultur + phänotyp. } \\
\text { Resistenztestung }\end{array}$ & $48(-72) \mathrm{h}$ & - alle Materialien & $\begin{array}{l}\text { Grundlage für } \\
\text { umfassende } \\
\text { Resistenztestung, } \\
\text { epidemiologische } \\
\text { Untersuchungen }\end{array}$ \\
\hline $\begin{array}{l}\text { Kultur + Identifizie- } \\
\text { rung + Resistenztes- } \\
\text { tung + PCR für PV- } \\
\text { Leukozidin }\end{array}$ & $48(-72) h$ & $\begin{array}{l}\text { - Anamnese } \\
\text { (rez. Abszesse etc.) } \\
\text { - MRSA mit isolierter } \\
\text { Resistenz gegen } \\
\text { Oxacillin } \pm \text { Fusidinsäure }\end{array}$ & \\
\hline
\end{tabular}

Methoden und dem Umfang der Probennahme bei verschiedenen Patienten findet sich in Tab.3.

Der Nachweis von Vancomycin-Resistenz gelingt nur unter Einsatz spezieller Testverfahren und deswegen nur nach Rücksprache des Klinikers mit dem mikrobiologischen Labor, wenn Patienten mit MRSA-Nachweis auf eine längere Therapie mit Glykopeptiden nicht ansprechen.

Molekulare Methoden zur Erstellung von genomischen Fingerabdrücken (Pulsfeld-Gelelektrophorese, Multilocus-Sequenzierung) haben viel zum Verständnis der Ausbreitung von MRSA beigetragen. Sie belegen die vorherrschende weltweite Verbreitung von einigen wenigen Stämmen („Epidemiestämme“), für die besondere Fähigkeiten zur Kolonisation und Infektion angenommen werden.

\section{Welcher Umfang der Diagnostik ist sinnvoll?}

Um einer weiteren Ausbreitung von MRSA in medizinischen Einrichtungen in Deutschland entgegenzutreten, ist eine möglichst frühzeitige Erkennung von Patienten mit Trägerstatus oder Infektion notwendig. Nach eigenen Untersuchungen beträgt im Falle eines unerkannten Bettnachbars mit MRSA das tägliche Risiko ca. $1 \%$, diesen Keim ebenfalls zu tragen bzw. zu erwerben. Umfangreiche und kostenintensive Isolierungsmaßnahmen machen nur Sinn, wenn das Fenster für eine Übertragung möglichst früh geschlossen wird. Ein Screening auf MRSA bei Aufnahme von Patienten wird als Bestandteil der „search \& destroy“-Politik in den Niederlanden erfolgreich eingesetzt ((14); siehe auch „Methicillin-resistenter Staphylococcus aureus (MRSA) - Therapie und Hygienemaßnahmen“). Zum Kosten-Nutzen-Verhältnis liegen eine Reihe von Studien vor $(10,14)$, die in Abhängigkeit von der Prävalenz für ein Aufnahmescreening auf MRSA sprechen. Dabei sollten bevorzugt moderne schnelle Methoden, wie z.B. PCR, eingesetzt werden. Aus hygienischen Gründen wäre ein Screening bei allen neu aufgenommenen Patienten wünschenswert, scheitert jedoch 
Tab.4 Indikation und Durchführung von MRSA-Screening-Untersuchungen.

\begin{tabular}{|c|c|c|c|}
\hline Personenkreis & Definition & Entnahme & Wiederholung \\
\hline $\begin{array}{l}\text { Risikopatienten } \\
\text { bei Aufnahme }\end{array}$ & $\begin{array}{l}\text { - MRSA bekannt* } \\
\text { - MRSA in } \\
\text { Vorgeschichte } \\
\text { - zuverlegt von } \\
\text { Intensivstation } \\
\text {-> } 14 \text { Krankenhaus- } \\
\text { tage im letzten Jahr }\end{array}$ & $\begin{array}{l}\text { - Nasenvorhof } \\
\text { bilateral + Wunde; } \\
\text { - ggf. + Leiste, } \\
\text { Rachen, Vagina }\end{array}$ & - \\
\hline $\begin{array}{l}\text { Kontakt- } \\
\text { patienten }\end{array}$ & $\begin{array}{l}\text { Im Zimmer mit } \\
\text { unerkanntem } \\
\text { MRSA-Patient }\end{array}$ & $\begin{array}{l}\text { - Nase bilateral } \\
\text { - Wunde } \\
\text { - ggf. + Leiste, } \\
\text { Rachen, Vagina }\end{array}$ & $\begin{array}{l}2 \times \text { wöchentlich } \\
\text { für die Dauer } \\
\text { des Aufenthalts }\end{array}$ \\
\hline $\begin{array}{l}\text { Sanierung des } \\
\text { Trägerstatus }\end{array}$ & $\begin{array}{l}\text { Bei vorherigem } \\
\text { Nachweis von MRSA; } \\
\text { aktuell keine MRSA- } \\
\text { wirksame Therapie }\end{array}$ & $\begin{array}{l}\text { - Nase bilateral } \\
\text { - Vorherige Herkunft } \\
\text { - ggf. + Leiste, } \\
\text { Rachen, Vagina }\end{array}$ & $\begin{array}{l}3 \times \text { negativ bis } \\
\text { zur Aufhebung } \\
\text { der Maßnahmen }\end{array}$ \\
\hline Personal & $\begin{array}{l}\text { Bei }>2 \text { neu erworbe- } \\
\text { nen Fällen auf } \\
\text { Station pro Woche }\end{array}$ & Nase bilateral & - \\
\hline \multicolumn{4}{|c|}{$\begin{array}{l}\text { *Durch Kennzeichnung der elektronischen Patientenakte wird die Indikationsstellung } \\
\text { wesentlich verbessert. }\end{array}$} \\
\hline
\end{tabular}

meist an den Kosten. Um die vorhandenen Ressourcen optimal einzusetzen, sollte MRSA-Screening bevorzugt bei Risikopatienten durchgeführt werden. Verbindliche Empfehlungen zur Definition von Risikopatienten, zur Anzahl der Proben, Methode etc. sind in Deutschland bislang nicht vorhanden. Tab.4 gibt einen Minimalkatalog, wie er für das Universitätsklinikum Regensburg verabschiedet wurde. Das Routinescreening wird mit nur einem Tupfer für beide Nasenvorhöfe durchgeführt. Falls vorhanden, sollte aus einer Wunde ebenfalls ein Abstrich erfolgen. Dieser Minimalansatz führt bei ca. 5-10\% der Patienten zu einem falsch-negativen Testergebnis, bei denen eine isolierte Besiedlung der Rachen- oder genitalen Schleimhäute vorliegt. Nur wenn bei einem Patienten MRSA gefunden wird, sollte der Kolonisationsstatus durch Abstriche der Nasenvorhöfe bilateral, sowie weiterer Prädilektionsstellen (Rachen, Leiste, Achsel, Vagina, Stuhl) bestimmt werden.

kurzgefasst: Der Nachweis von MRSA erfordert im Labor eine besondere Aufmerksamkeit. Die Art und Umfang der Diagnostik hängt von der jeweiligen diagnostischen Situation ab.

\section{Fazit}

Derzeit wird in Deutschland eine scheinbar ungebremste Zunahme von MRSA-Stämmen beobachtet. Mit der Ausbreitung auch außerhalb von Krankenhäusern ist in der Zukunft zu rechnen. Die Evolution von neuen MRSA-Stämmen mit neuartigen Kombinationen von Pathogenitäts- (PV-Leukozidin) oder Resistenzfaktoren (u.a. gegen Vancomycin) ist eine reale Bedrohung. Es bedarf erhöhter gemeinsamer Anstrengungen auf den Gebieten der Diagnostik, rationalem Antibiotikagebrauch, Standardhygiene und MRSA-spezifischen Hygienemaßnahmen, um diese Entwicklung umzukehren. Neu entwickelte schnelle diagnostische Verfahren sind als Screening-Instrumente zur möglichst frühen Identifizie- rung von Patienten mit MRSA geeignet und tragen dazu bei, dass die vorhandenen Ressourcen optimal eingesetzt werden können.

\section{Konsequenzen für Klinik und Praxis}

\section{- In Deutschland muss in Krankenhäusern zunehmend mit MRSA gerechnet werden \\ Bei Patienten mit rezidivierend auftretenden Abszessen ohne weitere Risikofaktoren kann als Ursache $S$. aureus mit PV-Leukozidin vorliegen \\ MRSA mit PV-Leukozidin kann auch außerhalb von me- dizinischen Einrichtungen erworben werden}

Autorenerklärung: LH und LN haben Unterstützung und Honorare für Vorträge von folgenden Firmen erhalten: Astra-Zeneca, Aventis, Bayer, Cubist, Dade-Behring, MSD, Pfizer, Roche, Schülke und Mayr.

\section{Literatur}

1 Becker K. Diagnostik von Methicillin-resistenten Staphylococcus aureus (MRSA)-Stämmen Teil 1. Taxonomische Einordnung, Anzucht und Differenzierung von Staphylococcus aureus. Mikrobiologe 2004; 14: 7-21

2 Becker K, Friedrich AW, Lubritz G et al. Prevalence of genes encoding pyrogenic toxin superantigens and exfoliative toxins among strains of Staphylococcus aureus isolated from blood and nasal specimens. J Clin Microbiol 2003; 41: 1434-1439

${ }^{3}$ Heuck D, Witte W. Methicillin-resistente Staphylococcus aureus (MRSA) in deutschen Alten- und Pflegeheimen - zur Situation. Epidemiologisches Bulletin 2003; 19: 145-148

${ }^{4}$ Höpgen ME, Dreesmann J, Braulke C et al. MRSA-Besiedlung in einem Alten- und Pflegeheim: Risikofaktoren und Prävalenz. Hygiene und Medizin 2001; 26: 225-230

${ }^{5}$ Kipp F, Friedrich AW, Becker K et al. Bedrohliche Zunahme Methicillin-resistenter Staphylococcus aureus-Stämme. Dt Ärztebl 2004; 101: 2045-2050

${ }^{6}$ Koning S, van Belkum A, Snijders S et al. Severity of nonbullous Staphylococcus aureus impetigo in children is associated with strains harboring genetic markers for exfoliative toxin B, Panton-Valentine leukocidin, and the multidrug resistance plasmid pSK41. J Clin Microbiol 2003; 41: 3017-3021

7 Lehn N, Linde H, Schneider-Brachert W et al. MRSA screening on admission by real time PCR directly from the swab within the same day. 14th Congress of the European Society of Clinical Microbiology and Infectious Diseases, Prag, 2004

8 Lina G, Piemont Y, Godail-Gamot $\mathrm{F}$ et al. Involvement of Panton-Valentine leukocidin-producing Staphylococcus aureus in primary skin infections and pneumonia. Clin Infect Dis 1999; 29: 1128-1132

${ }^{9}$ Naimi TS, LeDell KH, Boxrud DJ et al. Epidemiology and clonality of community-acquired methicillin-resistant Staphylococcus aureus in Minnesota, 1996-1998. Clin Infect Dis 2001; 33: 990-996

${ }^{10}$ Papia G, Louie M, Tralla A et al. Screening high-risk patients for methicillin-resistant Staphylococcus aureus on admission to the hospital: is it cost effective? Infect Control Hosp Epidemiol 1999; 20: 473-477

11 Prevost G, Couppie P, Prevost P et al. Epidemiological data on Staphylococcus aureus strains producing synergohymenotropic toxins. J Med Microbiol 1995; 42: 237-245

12 Safdar N, Maki DG. The commonality of risk factors for nosocomial colonization and infection with antimicrobial-resistant Staphylococcus aureus, enterococcus, gram-negative bacilli, Clostridium difficile, and Candida. Ann Intern Med 2002; 136: 834-844

13 Safdar N, Narans L, Gordon B et al. Comparison of culture screening methods for detection of nasal carriage of methicillin-resistant Staphylococcus aureus: a prospective study comparing 32 methods. J Clin Microbiol 2003; 41: 3163-3166

14 Vriens M, Blok H, Fluit A et al. Costs Associated with a Strict Policy to Eradicate Methicillin-Resistant Staphylococcus aureus in a Dutch University Medical Center: A 10-Year Survey. Eur J Clin Microbiol Infect Dis 2002; 21: 782-786 